\title{
Compton processes in the bright AGN MCG +8-11-11
}

\author{
Simona Soldi* \\ Laboratoire AIM - CNRS - CEA/DSM - Université Paris Diderot (UMR 7158), CEA Saclay, \\ DSM/IRFU/SAp, F91191 Gif-sur-Yvette, France \\ E-mail: simona.soldi@cea.fr
}

Volker Beckmann

François Arago Centre, APC, Université Paris Diderot, CNRS/IN2P3, CEA/DSM, Observatoire de Paris, 13 rue Watt, 75205 Paris Cedex 13, France

Neil Gehrels

NASA Goddard Space Flight Center, Astrophysics Science Division, Code 661, Greenbelt, MD 20771, USA

\section{Piotr Lubiński}

Nicolaus Copernicus Astronomical Center, Polish Academy of Sciences ul. Rabianska 8, 87-100 Torun, Poland

\section{Claudio Ricci}

ISDC Data Centre for Astrophysics, Observatoire de Genève, Chemin d'Ecogia 16, Versoix, Switzerland

\section{Roland Walter}

ISDC Data Centre for Astrophysics, Observatoire de Genève, Chemin d'Ecogia 16, Versoix, Switzerland

We present preliminary results on the hard X-ray emission properties of the Seyfert 1.5 galaxy MCG $+8-11-11$, as observed by INTEGRAL and SWIFT. All the INTEGRAL IBIS/ISGRI data available up to October 2009 have been analysed together with two SWIFT/XRT snapshot observations performed in August and October 2009, quasi-simultaneously to INTEGRAL pointed observations of MCG $+8-11-11$. No correlation is observed between the hard Xray flux and the spectral slope, while the position of the high-energy cut-off is found to have varied during the INTEGRAL observations. This points to a change in the temperature of the Comptonising medium from a minimum value of $k T_{\mathrm{e}}=30-50 \mathrm{keV}$ to values larger than 100$150 \mathrm{keV}$. There is no significant detection of Compton reflection, with a $3 \sigma$ upper limit of $R<0.2$, and no line has been detected at $112 \mathrm{keV}$, as previously claimed from HEAT observations $\left(F_{112 \mathrm{keV}}<2.4 \times 10^{-4} \mathrm{phcm}^{-2} \mathrm{~s}^{-1}\right)$. The variability behaviour of MCG $+8-11-11$ is found to be similar to that shown by IC 4329A, with different temperatures of the electron plasma for similar flux levels of the source, while other bright Seyfert galaxies present different variability patterns at hard X-rays, with spectral changes correlated to flux variations (e.g. NGC 4151).

8th INTEGRAL Workshop "The Restless Gamma-ray Universe"

September 27-30 2010

Dublin Castle, Dublin, Ireland

\footnotetext{
* Speaker.
} 


\section{Introduction}

The Seyfert 1.5 MCG $+8-11-11(z=0.0205)$ is one of the brightest Seyfert galaxies in the X-rays and historically showed flux variations by a factor of 3-4 on time scale of years (Fig. 1). This source has been extensively observed in the past below $10 \mathrm{keV}$, but only few observations were performed at hard X-rays before the advent of the present hard X-ray satellites INTEGRAL, SWIFT and Suzaku. Due to the relatively low amount of reflection and the properties of the Fe line, it has been suggested that the observed reflection is produced in the accretion disc and that the narrow core of the $\mathrm{Fe} \mathrm{K} \alpha$ line is emitted in the broad line region, whereas no evidences for a classical torus are found in the X-ray spectrum of this AGN [1], making MCG $+8-11-11$ a very peculiar Seyfert galaxy. We present here the preliminary results of simultaneous INTEGRAL [2] and SWIFT [3] pointed observations of MCG $+8-11-11$. Such observations aim at measuring the Comptonisation parameters for an increasing sample of Seyfert galaxies in order to test the AGN unification model and to provide more precise inputs for cosmic X-ray background models. In addition, the study of the spectral variability properties of AGN can provide important information about the structure, the physics and the dynamics of the radiating source.

\section{INTEGRAL and SWIFT observations}

Before the 2009 pointed observation, MCG $+8-11-11$ had been observed by INTEGRAL during non-dedicated observations, resulting in off-axis angles mostly larger than $7^{\circ}$. For this reason, a relatively low effective IBIS/ISGRI [4] exposure time of only $235 \mathrm{ks}$ had been accumulated, compared to the $660 \mathrm{ks}$ of field exposure. During August and October 2009, INTEGRAL has performed $400 \mathrm{ks}$ ( $242 \mathrm{ks}$ of effective, off-axis corrected ISGRI time) of pointed observations on MCG $+8-11-11$, coordinated with 2 quasi-simultaneous SWIFT snapshots of 2.4 and $3.6 \mathrm{ks}$, respectively. We have analysed all the IBIS/ISGRI data available in the archive with the source within

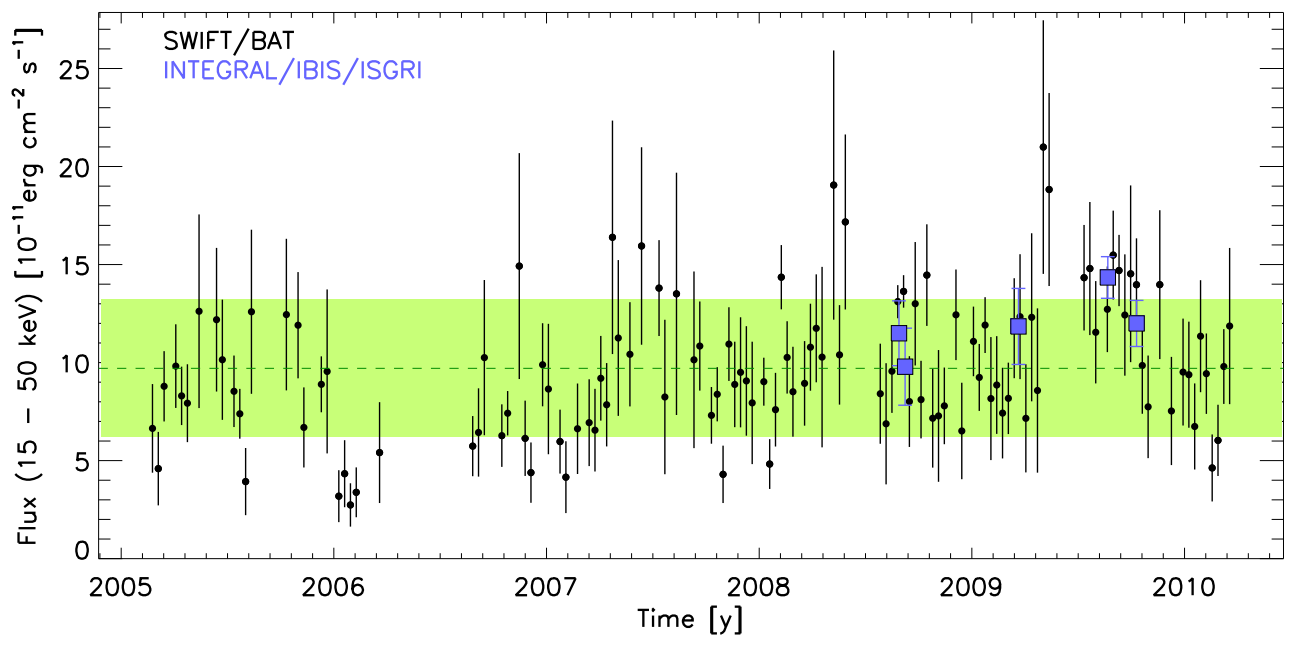

Figure 1: $\mathrm{MCG}+8-11-11$ light curve in the $15-50 \mathrm{keV}$ energy range as observed with SWIFT/BAT (black circles) and ISGRI (blue squares). The dashed line and the shaded area indicate the average value of the hard $\mathrm{X}$-ray flux measured by BAT and the dispersion from the mean. 


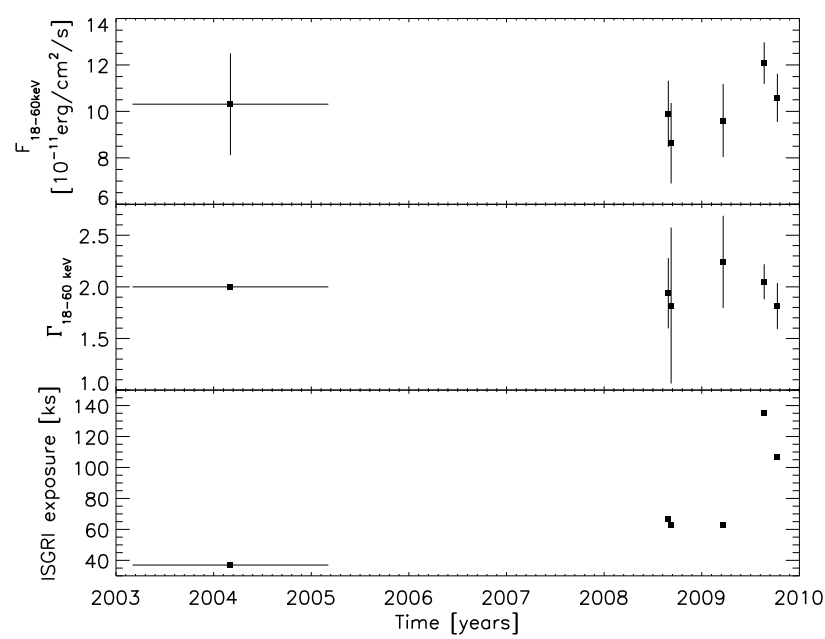

Figure 2: Evolution with time of the $18-60 \mathrm{keV}$ flux (top panel) and hard X-ray photon index (middle panel) as observed in ISGRI data. The bottom panel indicates the effective exposure time of the fitted spectrum. Due to low statistics, the photon index of the 2003-2005 spectrum has been fixed to a value of 2 .

$10^{\circ}$ from the center of the field of view, resulting in $477 \mathrm{ks}$ of effective exposure, accumulated between the beginning of the mission and October 11, 2009. The data have been analysed using version 9.0 of the Offline Scientific Analysis Software (OSA). The spectra have been extracted using the standard OSA spectral extraction.

The two SWIFT observations have been performed on August 26 and October 8, 2009 and the XRT data have been analysed with the SWIFT software version 3.4 distributed with the HEAsoft 6.7 package and the latest available calibration files.

\section{Spectral analysis}

\subsection{Spectral slope and flux variability}

We have grouped the IBIS/ISGRI data into 6 separate periods, spanning from 40 to $170 \mathrm{ks}$ of effective exposure time each, accumulated within 4 days to 2 years. In order to investigate the variations of the spectral shape and its correlation with the source flux across the mission lifetime, we have fitted these spectra with a simple power law model. The statistic of the spectrum collected during the first period (2003-2005) does not allow to constrain the power law slope, which has therefore been fixed to the value $\Gamma=2$. No correlation is found between the spectral slope and the 18-60 keV flux, with the flux varying by about $40 \%$ while the slope did not significantly change (Fig. 2). During the 2008-2009 INTEGRAL observations, the source was detected with a flux larger than its average level over the last 6 years as measured by SWIFT/BAT ${ }^{1}$ (Fig. 1).

\subsection{Comptonisation features}

A more detailed analysis has been carried out on the spectra with the highest-signal-to-noise, using a more complex model in order to test the presence of Comptonisation signatures, e.g. a

\footnotetext{
${ }^{1}$ The BAT light curve in the $15-50 \mathrm{keV}$ band has been downloaded from the Swift/BAT hard X-ray transient monitoring pages: http://swift.gsfc.nasa.gov/docs/swift/results/transients
} 

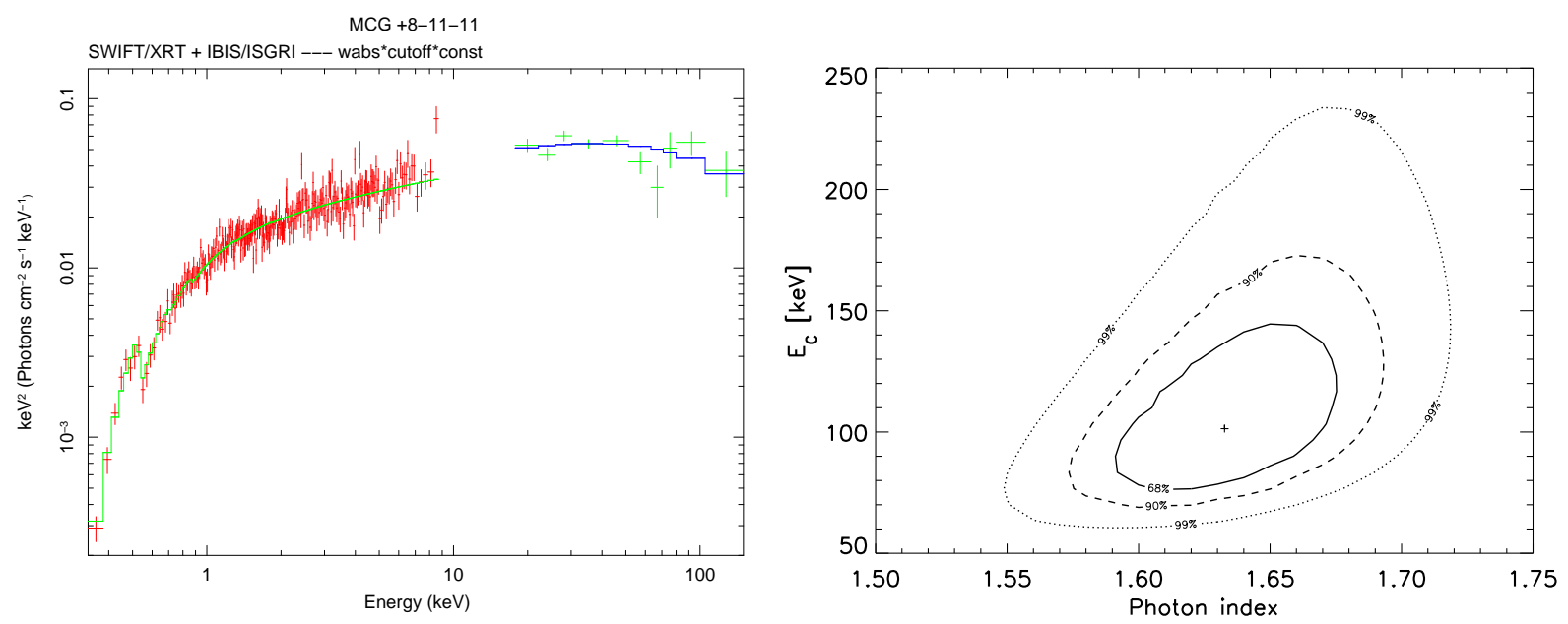

Figure 3: Left: Total INTEGRAL/IBIS ISGRI spectrum (spanning the whole mission time) and SWIFT/XRT averaged spectrum of MCG $+8-11-11$ fitted with an absorbed cut-off power law. Right: Cut-off energy versus photon index contour plot at $68 \%, 90 \%$ and $99 \%$ confidence level for the average XRT + total ISGRI spectra.

high-energy cut-off and a Compton reflection hump. Therefore, we fitted the IBIS/ISGRI spectra of August and October 2009 with the respective quasi-simultaneous SWIFT/XRT data, and the total ISGRI spectrum spanning 7 years of the INTEGRAL mission together with the average XRT spectrum. Preliminary results indicate that while the October 2009 spectrum is best fitted with a simple absorbed power law ( $\chi_{\text {red }}^{2}=0.93,174$ d.o.f.), the August 2009 spectrum shows a prominent curvature, best modeled with the addition of a cut-off at $E_{\mathrm{C}}=115_{-43}^{+117} \mathrm{keV}$, and resulting in a significant improvement of the reduced $\chi^{2}\left(\chi_{\text {red }}^{2}=0.99,126\right.$ d.o.f., F-test probability $\left.=4 \times 10^{-4}\right)$. Similarly, the total spectrum requires the addition of a cut-off at $E_{\mathrm{C}}=101_{-27}^{+48} \mathrm{keV}\left(\chi_{\text {red }}^{2}=1.0,265\right.$ d.o.f., F-test probability $=2 \times 10^{-8}$; Fig. 3). A summary of the best parameters obtained fitting these three ISGRI spectra with an absorbed cut-off power law model is provided in Table $1^{2}$. Only a $3 \sigma$ upper limit could be derived for the amount of Compton reflection present in the source ( $R<0.2$, when applying a PEXRAV model to the total spectrum) and for the flux of the Fe K $\alpha$ line $\left(F<3.1 \times 10^{-4} \mathrm{phcm}^{-2} \mathrm{~s}^{-1}\right.$, when fixing the rest frame line energy and the line width at $E_{\mathrm{Fe}}=6.4 \mathrm{keV}$ and $\sigma_{\mathrm{Fe}}=0.17 \mathrm{keV}$, respectively; [5]). We tried to fit the spectra with a more physical model, i.e. COMPPS, but it was not possible to significantly constrain its parameters. A more complete and detailed analysis of MCG +8-11-11 combining INTEGRAL, SWIFT, XMMNewton, RXTE and Suzaku data and using the COMPPS model is ongoing [6] and its preliminary results are in agreement with those presented here.

\subsection{The $112 \mathrm{keV}$ line}

An emission line at $112 \mathrm{keV}$ was detected in the spectrum of MCG $+8-11-11$ during a balloon flight of the HEAT telescope in July 1995 with a flux of $9 \times 10^{-4} \mathrm{phcm}^{-2} \mathrm{~s}^{-1}$ [7]. As simultaneously the veto system of the telescope registered a strong gamma-ray flux, Perotti et al.

\footnotetext{
${ }^{2} \mathrm{~A}$ cross-calibration factor has been added to all fitting models, and left free to vary. This factor has been found to assume values of $C=(0.8-0.9) \pm 0.1$ for the ISGRI spectra with respect to the XRT ones.
} 
have suggested that the $112 \mathrm{keV}$ line could be due to double-Compton scattering of a $511 \mathrm{keV}$ line source incident on an external cloud. Later CGRO/OSSE observations did not detect the line with an upper limit flux of $0.9 \times 10^{-4} \mathrm{ph} \mathrm{cm}^{-2} \mathrm{~s}^{-1}$ [8] implying that the line, if present, is variable. In the ISGRI data presented here, we do not detect any line at $112 \mathrm{keV}$. We provide a $3 \sigma$ upper limit of $2.4 \times 10^{-4} \mathrm{ph} \mathrm{cm}^{-2} \mathrm{~s}^{-1}$ for the line flux, when fixing the position and the width of the line to $E_{\text {line }}=112 \mathrm{keV}$ and $\sigma_{\text {line }}=32 \mathrm{keV}$ [7]. This upper limit is 4 times lower than the flux reported by Perotti et al. and consistent with the upper limit found with OSSE. The source was not detected by SPI and, in any case, due to its sensitivity and spectral resolution, ISGRI is a better instrument than SPI to detect a rather broad line at energies below $200 \mathrm{keV}$.

\section{Discussion and conclusions}

With a black hole mass of $M_{\mathrm{BH}}=(1.5-13) \times 10^{7} \mathrm{M}_{\odot}[9,10]$, an X-ray luminosity of $L_{0.1-300 \mathrm{keV}}$ $=2.7 \times 10^{44} \mathrm{erg} \mathrm{s}^{-1}$ and assuming a twice as large bolometric luminosity, $\mathrm{MCG}+8-11-11$ shows an Eddington ratio of $L_{\mathrm{Bol}} / L_{\mathrm{Edd}}=0.03-0.3$, placing it among the most efficient hard X-ray selected Seyfert galaxies [11].

Some of the past studies of MCG $+8-11-11$ reported the presence of a strong reflection component $R=1-2.5[5,8,12,13]$. Yet, a good statistical constrain was only obtained with $X M M$ Newton data but limited at energies below $10 \mathrm{keV} \mathrm{[5]} \mathrm{or} \mathrm{combined} \mathrm{with} \mathrm{low} \mathrm{effective} \mathrm{exposure}$ ISGRI data ([13], corresponding to the first point in Fig. 2), until Suzaku together with SWIFT/BAT observations provided a high signal-to-noise, broad band coverage between 0.6 and $150 \mathrm{keV}$ [1]. The latter work finds that when the broad band spectrum is fitted, the reflection fraction drops to values of $R=0.2-0.3$, marginally consistent with the upper limit we find in INTEGRAL data.

During observations at hard X-rays with CGRO/OSSE [8], BeppoSAX [12], Suzaku + Swift/BAT [1] and in early INTEGRAL/ISGRI data [13], MCG $+8-11-11$ showed a somewhat steeper spectrum $(\Gamma=1.7-1.9)$ and a cut-off at higher energies $\left(E_{\mathrm{C}}=130-270 \mathrm{keV}\right)$, but still consistent within the uncertainties with the value found here for the August 2009 spectrum. A cut-off energy at $\sim 100 \mathrm{keV}$ corresponds to an electron plasma temperature of $k T_{\mathrm{e}}=30-50 \mathrm{keV}$, towards the lower end of typical values found in Seyfert galaxies [14]. INTEGRAL data seem to indicate that the position of the high-energy cut-off varies on month time scale, while the spectral slope and the flux did not significantly change during the same period. Yet, it is important to keep in mind the uncertainties of the cut-off determination, due to the fact that the source is detected up to only $\sim 120 \mathrm{keV}$ in these ISGRI data.

Past studies suggest that the hard X-ray emission of Seyfert galaxies is indeed observed to vary, and the variations can be complex and very different from object to object. For instance, in some

\begin{tabular}{ccccccc}
\hline \hline $\begin{array}{c}\text { ISGRI obs. } \\
\text { period }\end{array}$ & $\begin{array}{c}\text { ISGRI exp. } \\
{[\mathrm{ks}]}\end{array}$ & $\begin{array}{c}N_{\mathrm{H}} \\
{\left[10^{22} \mathrm{~cm}^{-2}\right]}\end{array}$ & $\Gamma$ & $\begin{array}{c}E_{\mathrm{C}} \\
{[\mathrm{keV}]}\end{array}$ & $\begin{array}{c}F_{2-10 \mathrm{keV}} \\
\mathrm{erg} \mathrm{cm}^{-2} \mathrm{~s}^{-1}\end{array}$ & $\begin{array}{c}F_{20-60 \mathrm{keV}} \\
\mathrm{erg} \mathrm{cm}^{-2} \mathrm{~s}^{-1}\end{array}$ \\
\hline August 2009 & 135 & $0.21 \pm 0.03$ & $1.61 \pm 0.08$ & $115_{-43}^{+117}$ & $8.1 \times 10^{-11}$ & $1.1 \times 10^{-10}$ \\
October 2009 & 173 & $0.18 \pm 0.02$ & $1.69 \pm 0.06$ & $\geq 320$ & $6.6 \times 10^{-11}$ & $9.6 \times 10^{-11}$ \\
All data & 477 & $0.19 \pm 0.01$ & $1.63 \pm 0.05$ & $101_{-27}^{+48}$ & $7.2 \times 10^{-11}$ & $9.4 \times 10^{-11}$ \\
\hline
\end{tabular}

Table 1: Best fit parameters for the XRT+ISGRI spectra of MCG $+8-11-11$. 
cases no spectral variations are observed to accompany different flux levels [15] whereas in some other cases variations of the spectral slope are correlated with the flux [16], suggesting the superposition of a constant reflection component and a variable (in flux and shape) power law continuum. On the other hand, NGC 4151 presents no changes in the slope of the underlying continuum for different X-ray fluxes but a significant variation of the temperature of the Comptonising plasma is observed [14]. For other objects, a clear variability pattern has not been recognized yet and equivalent flux levels can present significantly different spectral slopes (NGC 4388 [17], NGC 7172 [18]) or different cut-off energies (this work and IC 4329A [19]).

The large amount of INTEGRAL data currently available will enable us to extend this kind of studies to about 20 Seyfert galaxies, among which NGC 7172, NGC 4593, NGC 2110, Mrk 509 [20], as already done for other INTEGRAL AGN (e.g. [14, 17, 21, 22, 23, 24]).

\section{Acknowledgments}

S.S. acknowledges the Centre National d'Etudes Spatiales (CNES) for financial support. Part of the present work is based on observations with INTEGRAL, an ESA project with instruments and science data centre funded by ESA member states with the participation of Russia and the USA. We thank Peter Kretschmar for providing the script to draw the contour plot.

\section{References}

[1] Bianchi S., De Angelis I., Matt G., et al. 2010, A\&A, 522, A64

[2] Winkler C., Gehrels N., Schönfelder, V., et al. 2003, A\&A, 411, 349

[3] Gehrels N., Chincarini G., Giommi P., et al. 2004, ApJ, 611, 1005

[4] Lebrun F., Leray J. P., Lavocat P., et al. 2003, A\&A, 411, 141

[5] Matt G., Bianchi S., De Rosa A., Grandi P., Perola G. C. 2006, A\&A 445, 451

[6] Lubiński P., Beckmann V., Paltani S., et al. in preparation

[7] Perotti F., Mattaini E., Quadrini E., et al. 1997, ApJ 475, L89

[8] Grandi P., Haardt F., Ghisellini G., et al. 1998, ApJ 498, 22

[9] Bian W. \& Zhao Y. 2003, MNRAS 343, 164

[10] Middleton M., Done C., Schurch N. 2008, MNRAS 383, 1501

[11] Beckmann V., Soldi S., Ricci C., et al. 2009, A\&A, 505, 417

[12] Perola G.C., Matt G., Fiore F., et al., 2000, A\&A 358, 117

[13] Molina M., Bassani L., Malizia A., et al. 2009, MNRAS 399, 1293

[14] Lubiński P., Zdziarski A. A., Walter R., et al. 2010, MNRAS 408, 1851

[15] Guainazzi M., Perola G. C., Matt G., et al. 1999, A\&A 346, 407

[16] Papadakis I.E., Sobolewska M., Arevalo P., et al. 2009, A\&A 494, 905

[17] Fedorova E.V., Beckmann V., Neronov A., Soldi S. 2011, PoS(INTEGRAL2010)075

[18] Guainazzi M., Matt G., Antonelli L.A., et al. 1998, A\&A 298, 824

[19] Soldi S., Ponti G., Beckmann V., Lubiński P. 2009, PoS(extremesky2009)031

[20] Paltani S., Petrucci P.O., Lubiński P., et al. 2011, PoS(INTEGRAL2010)009

[21] Beckmann V., Courvoisier T. J.-L., Lubiński P., et al. 2008, A\&A, 492, 93

[22] Beckmann V., Jean P., Lubiński P., Soldi S., Terrier R. 2011, submitted to A\&A

[23] Soldi S., Beckmann V., Bassani L., et al. 2005, A\&A 444, 431

[24] Beckmann V. \& Do Cao O. 2011, PoS(INTEGRAL2010)081 